\title{
Proposta de um Programa para a Formação do Residente em Medicina de Família e Comunidade
}

\section{A Proposal for a Family Medicine Residency Program}

Carlos Arteaga Rodríguez André Lúcio de Cassias Marcelo Garcia Kolling ${ }^{1}$

\section{RESUMO}

É imperativa a formação de um Residente de Medicina de Família e Comunidade (RMFC) que responda às demandas da saúde pública, porém consideramos que ainda não existe uma concordância no programa para sua formação. Realizou-se uma pesquisa teórica para delinear a proposta de programa para a formação do RMFC embasada na revisão da literatura, nas recomendações da Comissão Nacional de Residência Médica e Sociedade de Medicina de Família e Comunidade do Brasil e na opinião dos RMFC da Pontifícia Universidade Católica do Paraná (PUC-PR), copilada pelo autor principal durante cinco anos como preceptor dos RMFC da PUC-PR. O programa inclui atividades práticas, teórico-práticas, investigativas e avaliativas desenvolvidas durante dois anos na Unidade de Saúde e no Hospital Regional, integrando o processo docente ao assistencial mediante a formação em serviço e no trabalho conjunto da faculdade com a Secretaria Municipal de Saúde e população, com uma visão preventivo-curativa e biopsicossocial do processo saúde-enfermidade, em função da problemática do indivíduo, da família e comunidade. Acreditamos que a proposta possa contribuir para o debate deste tema complexo e polêmico, e que, entre os acertos e desacertos dos programas, encontremos um consenso para a formação do médico de família e comunidade que o Brasil necessita.

\section{KEY-WORDS:}

-Medical Education;

-Family Medicine Residency;

-Family Health Program.

Recebido em: 30/09/2005

Reencaminhado em: 21/04/2007

Reencaminhado em: 27/07/2007

Reencaminhado em: 03/08/2007

40 REVISTA BRASILEIRA DE EDUCAÇÃO MÉDICA

\section{ABstract}

The training of family practitioners is essential for meeting the extensive health care demand of the Brazilian With the purpose of delineating a proposal for such a program a theoretical study was conducted taking as a basis a review of the literature, recommendations of the Comissão Nacional de Residência Médica, Sociedade Brasileira de Medicina de Familia e Comunidade and the opinion of family medicine residents of the Pontificia Universidade Católica of Paraná. The data were gathered over five years by the main author of this article and preceptor of the family health residents at the mentioned university. The program includes medical knowledge, practice-based learning, systems-based practice, research and evaluation, to be developed for two years in primary and secondary care facilities. Community assistance is combined with teaching through in-service training and cooperation of the medical school, the local health authorities and the population, building a preventive-curative biopsychosocial paradigm of the health-disease process focused on the individual, the family and the community. We believe that this proposal can contribute to the discussion about this complex and polemic subject and that it will be possible to reach a consensus with regard to the training of the family practitioners needed in Brazil. population, however there is still no consensus about a Family Medicine Residency Program in this field. 


\section{INTRODUÇÃO}

É óbvio o impacto do desenvolvimento técnico-científico, porém considerá-lo fonte de problemas éticos, religiosos, econômicos, biológicos, psicológicos e médicos merece reflexão. No caso da medicina, o curandeiro surgiu em estreito contato com o ser humano, e, mais tarde, o conhecimento fez surgir a medicina como ciência, representada no século 18 pela Clínica Geral e posteriormente pelas numerosas especialidades médicas ${ }^{1}$. Este desenvolvimento levou a classe médica a subestimar a atenção primária, o trabalho em equipe, os aspectos preventivos e generalistas, e a valorizar uma prática especializada, fragmentária, curativa, tecnicista e hospitalar-4.

Para Capra², "o sistema de assistência médica moderno está baseado no atendimento hospitalar e no uso de drogas, sendo incapaz de melhorar ou manter a saúde pública". Costa Neto $^{5}$ nos disse:

O cientificismo excessivo atual das práticas de saúde, desvinculado do humanismo e da ética, levou o setor a uma linha de ação cuja ligação entre o profissional e o usuário do sistema passou a ser muito mais o exame e o equipamento, em detrimento do relacionamento pessoal e profissional.

Então, a especialização favoreceu a elevação dos custos médi$\cos$, o menosprezo da relação médico-paciente e da confecção do prontuário médico e a fragmentação do doente, que, para tratar a diabetes mellitus com suas complicações, precisa consultar vários especialistas $^{6-12}$.

No Brasil, que necessita de maior número de médicos generalistas, essa situação é inadmissível e se agrava pelo desinteresse dos acadêmicos e médicos recém-formados pelo estudo e prática generalista e pela atenção primária, situação preocupante também nos países desenvolvidos. Nos Estados Unidos, as vagas para especialidades generalistas têm ocupação de 50 a $60 \%$, das quais $30 \%$ são abandonadas. Dos que não as abandonam, $60 \%$ voltamse para outra especialidade, $21 \%$ não voltariam a fazer esta especialidade, e 19\% escolheriam outra profissão não médica ${ }^{11}$. Cleaveland ${ }^{13}$, referindo-se ao desinteresse pelo generalismo nos Estados Unidos, comenta: "isto é terrível para um país que necessita de mais e não de menos médicos para a atenção primária".

Esta práxis explicaria, em parte, as listagens intermináveis do Sistema Único de Saúde para consultar o especialista e a insatisfação da comunidade com o atendimento especializado e na Unidade de Saúde (US). É o Médico de Família e Comunidade (MoFC) que está chamado a resolver esta problemática, gerada pelo desenvolvimento, por sua visão integradora dos aspectos biológicos, psicológicos e sociais em torno da saúde-enfermidade, por executar ações de educação, promoção, prevenção e por suas possibilidades objetivas de resolver a maioria dos problemas médicos, cifras que vão desde 80 até $90 \% \%^{14}$.

A despeito desta convicção, poucas universidades brasileiras incluem em seus cursos esta Residência Médica. Por outro lado, embora o Programa de Saúde da Família (PSF) ${ }^{15}$ esteja implantado há cerca de 30 anos no Brasil, não se conseguiu sensibilizar os egressos a direcionar sua pós-graduação para esta residência emergente, pois preferem outras, mais diferenciadas ${ }^{16-18}$. Assinale-se também que existem poucas publicações no Brasil acerca dos Programas de Residência de Medicina de Família e Comunidade (PRMFC), encontrando-se só um artigo na Revista Brasileira de Educação Médica18.

Os primeiros programas de formação de médicos para atuação em comunidades surgiram em 1976 no Rio Grande do Sul, Rio de Janeiro e Pernambuco. A trajetória da especialidade e da especialização em Medicina de Família e Comunidade (MFC) se fez cheia de encontros e desencontros, movendo-se, em muitos momentos, sob a direção das propostas do Ministério da Saúde. Hoje, existem cerca de 40 PRMFC $^{19}$. Além disso, a Comissão Nacional de Residência Médica (CNRM) ${ }^{20}$ tem normas que determinam os requisitos mínimos para os PRMFC, mas ainda não existe um consenso quanto à melhor maneira de se formar o Residente de Medicina de Família e Comunidade (RMFC) e qual currículo e forma de aplicação o residente deve seguir. Um caminho se vislumbra com a publicação recente de um documento pela Sociedade Brasileira de Medicina de Família e Comunidade (SBMFC) junto com o Ministério de Saúde (MS) ${ }^{21}$.

Neste panorama, pretendemos oferecer uma proposta de programa para a formação do RMFC inserido no PSF e onde se integra o processo formativo ao assistencial por meio da formação em serviço, entrelaçando a Faculdade de Medicina com os órgãos responsáveis pela saúde e pela formação do residente (Figura 1).

\section{METODOLOGIA}

Realizou-se um ensaio teórico-reflexivo, que compreende a descrição, registro, análise e interpretação do processo por meio da comparação e do contraste, baseado na argumentação e interpretação pessoal22,23, visando caracterizar e estruturar uma proposta de PRMFC. Aliando o método dedutivo à técnica de pesquisa bibliográfica, realizou-se um levantamento bibliográfico parcial nas bases de dados Medline, SciELO e Bireme, bem como nas bibliotecas da Universidade Federal do Paraná e da Pontifícia Universidade Católica do Paraná (PUC-PR).

A composição e a estrutura do programa proposto estão embasadas na revisão da literatura científica acerca do tema, nas mudanças do ensino universitário e médico, nas recomendações 
da CNRM $^{20}$ e da SBMFC21, na experiência de mais de cinco anos do autor principal como preceptor da RMFC e nos julgamentos dos assinantes do artigo, dois deles ex-residentes de MFC da PUC-PR. Mantém-se a ponte entre as propostas de mudanças na graduação médica com a formação do MoFC, preservando-se o princípio de continuidade entre os cursos de graduação e pós-graduação.

\section{DO PROGRAMA}

O programa sugere o vínculo entre os responsáveis pela formação do residente, Faculdade de Medicina, e os responsáveis pela assistência médica à população, Secretaria Municipal de Saúde (SMS), para assim cumprir o propósito da Educação Médica de relacionar o ensino à assistência e com os órgãos gestores da saúde em função da saúde da população ${ }^{24-27}$. Considera-se que a formação do residente deve ser responsabilidade da faculdade como requisito indispensável da educação continuada e entre a graduação e a pós-graduação, assuntos discutidos desde a 1ª Conferência Mundial de Educação Médica em 198826. Todavia, a SMS deve compreender o processo, para evitar o assistencialismo em detrimento da qualidade da assistência/formação, e os residentes e preceptores devem se conscientizar da necessidade de cobertura médica da população. Propõe-se uma estrutura onde as US estejam ligadas a um Hospital Regional (HR) com possibilidades de encaminhamento para serviços de maior complexidade, desenhada para ser adaptável ao contexto de diferentes municípios (Figura 1).

O programa enfatiza suas atividades instrutivas e educativas no trabalho, sem menosprezar o valor do conhecimento teórico, levando em consideração que prática e teoria são inseparáveis, de forma que o conhecimento transcorra da teoria à prática e da prática para a teoria ${ }^{28-30}$, com dois anos de duração. Da carga horária total (CHT), 10\% serão destinados a atividades teóricas e $90 \%$ a atividades de treinamento em serviço (ATS). Destas, 60\% serão em atenção primária e 40\% em atenção secundária, seguindo as orientações da $\mathrm{CNRM}^{20}$ e SBMFC ${ }^{21}$. As atividades estão direcionadas ao cuidado médico individual, familiar e comunitário, considerando uma abordagem biopsicossocial do processo saúde-enfermidade, realizando de forma integral ações de promoção, proteção e prevenção, e ações curativas e de reabilitação nos diferentes grupos etários e níveis de atenção médica (Figura 1).

Atendendo às necessidades de saúde no Brasil, o MoFC deve ser capaz de diagnosticar e tratar as principais doenças com as quais vai lidar, daí o propósito de que adquira as competências das chamadas Especialidades Básicas (Clínica Médica, Pediatria, Ginecologia-Obstetrícia e Cirurgia Geral) para oferecer uma assistência médica de qualidade. No entanto, necessita ainda domí- nio das ações de promoção, prevenção e educação da saúde individual, familiar e comunitária.

Para garantir o cumprimento do programa, são necessárias reuniões técnicas e administrativas mensais com os residentes, docentes, chefes de enfermagem, representantes da SMS, da Comissão de Residência Médica e da comunidade em que se discutam o processo docente e assistencial e a problemática de saúde local, para planejar as atividades de prevenção, promoção e proteção à saúde. Também devem ser realizadas jornadas científicas internas para discutir o andamento de trabalhos de pesquisa com vistas a seu aperfeiçoamento, para apresentação e publicação final. Estas jornadas facilitariam a atualização e a discussão de temas polêmicos e de interesse prático para a pesquisa.

A fim de otimizar o atendimento médico e a utilização dos exames complementares, é importante que exista um registro informatizado dos prontuários, acessíveis em qualquer dos níveis de atenção médica. Fica implícita a responsabilidade dos médicos na elaboração correta destes e a necessidade de que as SMS e as escolas médicas garantam material para estes fins.

\section{DOS OBJETIVOS DO PROGRAMA}

A primeira tarefa é mudar a concepção que o residente traz da graduação acerca da saúde e doença, sua visão hospitalocêntrica e tecnicista, com ações curativas e centradas na atenção do doente por especialidade, desvinculadas das ações preventivas e educativas da comunidade, da família e do indivíduo, elementos incorporados em currículos de várias universidades ${ }^{31,32}$.

Geral: proporcionar o aperfeiçoamento acadêmico, assistencial e investigativo do futuro MFC, fortalecer seu raciocínio clínico, criatividade, autonomia e interdependência, para que, ao término da residência, desenvolva seu trabalho no Sistema de Saúde Pública, Medicina de Grupo ou Prática Médica Privada.

Específicos: 1. Função Assistencial e Integradora: desenvolver uma visão integral da medicina em suas missões preventivas e curativas, em que os aspectos biológicos, psicológicos e sociais estão integrados, inter-relacionados e interdependentes. Interiorizar sua responsabilidade no atendimento dos pacientes para preservar e promover a saúde, preservar a vida e aliviar o sofrimento. 2. Função Educativa e Preventiva: desenvolver suas capacidades para persuadir, educar, instruir e assessorar corretamente o indivíduo, os familiares e a comunidade. 3. Função Social: compreender, valorizar e atuar sobre os fenômenos econômico-sociais e o meio onde habitam as pessoas. 4. Função Gerencial: desenvolver sua capacidade de gerência dos recursos humanos e materiais. 5. Função Científica: facilitar a utilização correta do Método Clínico como premissa na abordagem humana e profissional de seu doente, e com o propósito de otimizar e racionalizar o uso dos exames complementares e evitar a polifarmácia injustificada. 6 . 
Função Interdisciplinar: encaminhar e discutir com outras especialidades médicas quando as circunstâncias assim o justifiquem. 7. Função Legal: exercer criteriosamente sua atividade profissional, para evitar a imperícia, imprudência e negligência, assim como sua responsabilidade e obrigações legais na emissão de documentos e certificados. 8. Função Ética: manter uma conduta ética no relacionamento com seus pacientes e familiares, seus colegas e com outros profissionais, segundo os postulados do Código Brasileiro de Ética Médica. 9. Função Autopreparatória: favorecer o hábito de autopreparação e atualização continuada.

\section{DOS PROFESSORES/PRECEPTORES}

Os professores/preceptores (PP) são responsáveis pela supervisão e orientação do residente, relação fundamental para garantir uma assistência e formação médica de qualidade, ao mesmo tempo em que são exemplos para o residente, expectativas comentadas por Lopez et al. ${ }^{33}$. Devem ser reconhecidos por sua preparação científica e técnica, humanismo, dedicação e preparação docente ${ }^{34}$.

Para o trabalho da atenção primária, propõe-se um PP especialista em MFC que supervisione as consultas do residente na US e nas atividades de área, além de orientar o trabalho de gerenciamento e tutorar as pesquisas. Considera-se uma proporção de um PP para cada dois residentes. Para o trabalho no HR, quatro PP, um por especialidade-Clínica Médica, Gineco-Obstetricia, Cirurgia Geral e Pediatria -, que supervisionem os residentes nas atividades de Enfermaria, Pronto Socorro, Centro Cirúrgico e Centro Obstétrico. Para garantir a integração das quatro especialidades básicas, existirá PP especialista em MFC. Os PP do HR realizarão interconsultas periódicas na US, para discussão e orientação dos casos complexos.

Para coordenar as atividades na atenção primária e secundária e para estabelecer o nexo entre as instituições assistenciais e docentes, é imprescindível a existência de um coordenador especialista em MFC.

No curso de 2005 da Residência da PUC-PR, temos convidado professores de outras especialidades, como Cardiologia, Psiquiatria e Neurologia, para oferecerem temas de atualização sobre problemas freqüentes e de interesse para o trabalho do MFC, escolhidos pelos próprios residentes, como eletrocardiograma, demências, depressão, uso de psicofármacos e manejo da dor, entre outros, com excelente aprovação pelos residentes.

\section{DAS ATIVIDADES DOCENTES E ASSISTENCIAIS}

Estão distribuídas em práticas, teórico-práticas, investigativas e avaliativas. As práticas e teórico-práticas estão direcionadas ao cuidado médico individual, familiar e comunitário, levando em conta uma abordagem biopsicossocial do processo saúde-enfer- midade. Estas atividades estão concebidas para serem desenvolvidas na US e no HR, integrando estes dois níveis de atenção primária e secundária - entre si e com o nível terciário.

O RMFC, supervisionado pelo PP, fará uma abordagem integral do paciente, compreendendo-o dentro de seu ciclo de vida, de sua estrutura e dinâmica familiar, sendo capaz de identificar transtornos adaptativos e de aplicar os princípios de psicoterapia, orientando de forma racional os exames complementares e sendo capaz de interpretá-los. Desenvolverá ações de caráter multidisciplinar e interdisciplinar, apoiando as chefias nos trabalhos gerenciais e integrando-se a outros profissionais num trabalho em equipe, que é uma característica da medicina de família ${ }^{35}$.

No primeiro ano, o residente desenvolverá atividades na US, onde se integrará à equipe do PSF, realizando trabalho de área e de consultório, segundo as recomendações do MS15. Além disso, dará plantões no HR. O primeiro mês será destinado à familiarização na US e no HR (Quadro 1).

O trabalho de área inclui visitas domiciliares para acompanhamento de pacientes crônicos, complexos, acamados ou internamentos domiciliares, visitas após alta hospitalar, investigação de pacientes faltosos ao consultório, palestras de educação em saúde, educação das famílias de risco, trabalho com grupos como hipertensos, diabéticos, gestantes e puericultura, entre outros. Como parte do PSF, desempenhará tarefas de coordenação com os órgãos políticos, administrativos e instituições da comunidade.

Propõe-se que o RMFC, em atividade de consultório, desenvolva, planeje, execute e avalie programas integrais da saúde da criança e do adulto, como: Programas de Atenção para os Grupos de Risco (lactentes, crianças com baixo peso, adolescentes e idosos); Programas de Atenção para Doenças Crônicas Transmissíveis (tuberculoses, hanseníase, doenças sexualmente transmissíveis e outras) e não transmissíveis (hipertensão arterial sistêmica, diabetes mellitus, doenças cerebrovasculares, obesidade, carências nutricionais, transtornos mentais); Programa de Atenção Integral à Mulher (acompanhamento da gestação e puerpério normais, identificação dos níveis de risco gestacional, manejo das afecções mais freqüentes do ciclo gravídico-puerperal e do climatério, prevenção de câncer de mama e de colo uterino); Programa de Planejamento Familiar (orientação para adolescentes sobre métodos contraceptivos); Programa de Imunização e Vigilância Epidemiológica.

Junto com sua equipe, confeccionará um registro, por área, dos pacientes por grupos etários, casos com doenças crônicas, surtos de doenças agudas infectocontagiosas, casos complexos, doenças ocupacionais, esquema de vacinação e cumprimento terapêutico das enfermidades crônicas transmissíveis que facilite o controle e pesquisa clínico-epidemiológica da US. Outras tarefas incluem: confecção do prontuário e arquivo médico; realização de 
consultas para diagnóstico e tratamento das doenças da infância, adolescência, adulto e idoso, reconhecimento dos distúrbios psicológicos ehábitos tóxicos; primeiros cuidados às afecções graves eurgentes antes doencaminhamento; pequenas cirurgias; notificação das doenças de declaração obrigatória; preenchimento correto dos pedidos de exames, atestados médicos, de óbito e encaminhamentos para outras especialidades.

No segundo ano, desenvolverá atividades no HR pela manhã e, noperíodo da tarde, integrarána USas atividades docente-assistenciais. As atividades na Atenção Secundária objetivam que o RMFC, nas Enfermarias, Centro Cirúrgico, Obstétrico e Pronto Socorro do HR, adquira a competência necessária para seu desempenho no atendimento clínico, pediátrico, assistência ao parto e cuidados do puerpérioe do recém-nascido, reconhecimento das afecções cirúrgicas para seu encaminhamento, realização de pequenas cirurgias e orientação de pré e pós-operatório. Um mês será destinado a um estágio eletivo em outras instituições (Quadro 1). Este estágio permite que o RMFC traga experiências a serem discutidas em grupo, o que, pela nossa experiência, representa uma fonte de retroalimentação para o PRMFC.

Por ocasião da alta hospitalar, será realizada uma rediscussão que inclua a revisão do diagnóstico e exames complementares, oferecendo ao paciente e familiares as orientações pertinentes em relação ao atendimento hospitalar, além das condutas terapêuticas e acompanhamento na US. Serão entregues, junto com o resumo de alta por escrito, as receitas médicas legíveis, com remédios que possam ser adquiridos. Tanto na US como no HR se fará análise de óbitos, e, quando viável, recomendamos a realização da autópsia e discussão de peças em fresco.

A importância da pesquisa na formação do MoFC tem sido abordada ${ }^{36,37}$. Tais pesquisas pretendem promover estudos sobre incidência e prevalência, morbi-mortalidade e letalidade nos diferentes grupos etários, indicadores de saúde e condições epidemiológicas da comunidade; investigar novas técnicas na assistência da MFC; criar programas para a atenção integral à criança, adolescente, mulher, adulto e idoso saudáveis, bem como programas de controle e atenção às doenças crônicas transmissíveis e não transmissíveis; padronizar condutas segundo os critérios da medicina baseada em evidências (MBE); desenvolver métodos para avaliar o grau de cumprimento, eficácia, eficiência e efetividade dos programas.

A pesquisa estará assim distribuída: no primeiro semestre, confecção do projeto; no segundo e terceiro, coleta de dados; no quarto, confecção e apresentação dos resultados. Uma vez concluída, deverá ser apresentada e publicada. Consideramos que a conclusão da pesquisa deve ser condicionante para a obtenção do título de especialista e fazer parte da avaliação dos preceptores tutores.

As atividades teóricas serão realizadas conjuntamente com os RMFC do primeiro e segundo anos. Para facilitar a aquisição de conhecimento, recomenda-se elaborar um programa analítico embasadona problemática de saúdeda populaçãoabrangida, levando em consideração as recomendações dos preceptores, os temas que os residentes julguem de maior complexidade e interesse, os temas próprios da MFC, as estimativas dos serviços de vigilância epidemiológica ${ }^{38}$ e temas a serem debatidos nas atividades docentes de grupo e como estudo individual.

Para a abordagem dos temas, propõem-se o ensino baseado em problemas e a $\mathrm{MBE}^{39-41}$, utilizando-se as seguintes formas de organização docente: visitas às enfermarias, consultas médicas domiciliares e na unidade de saúde, discussão de casos clínicos, seminários, mesas-redondas e conferências (Quadro 2). Nelas, os residentes são os responsáveis pela apresentação dos temas supervisionados pelo PP, o que facilita sua aprendizagem e desenvolvimento na comunicação oral, elementos importantes para seu trabalho e como professores potenciais. Recomenda-se realizar, uma vez por mês, uma discussão de caso que integre todas as áreas de atuação.

Como fonte teórica, sugerimos a seguinte bibliografia:

MFC: Duncan, B. B. et al. Medicina ambulatorial: condutas de atenção primária baseadas em evidências. 3. ed. Porto Alegre: Artes Médicas Sul, 2004. Rakel, R. E. Tratado de Medicina de Família. 6. ed. Rio de Janeiro: Guanabara Koogan, 2002. American Family Physician. Family Practice Management. Annals of Family Medicine. Canadian Family Medicine. Archives of Family Medicine. Caderno de Saúde Pública. Revista de Saúde Pública e Fundação Nacional de Saúde (Funasa-MS). Revista Cubana de Medicina General e Integral. CLÍNICA MÉDICA: Postgraduated Medicine. Cecil, R. L. et al. Cecil Medicina Interna. 22. ed. Rio de Janeiro: Guanabara Koogan, 2005. PEDIATRIA: Nelson Tratado de Pediatria. 17 ed. Rio de Janeiro: Guanabara, 2005. Hisler, H. et al. Pediatria na Atenção Primária. São Paulo: Savier, 1999. American Heart Association. Suporte Avançado deVida em Pediatria. F.G.Stoddard, 1999. GINECO-OBSTETRÍCIA: Halbe, H. W., Berman, R. E. et al. Tratado de Ginecologia. 3. ed. São Paulo: Rocca, 2004. Rezende, J. de et al. Obstetrícia Fundamental. 8. Ed. Rio de Janeiro: Guanabara Koogan, 1999. CIRURGIA GERAL: Sabiston, D. C. et al. Sabiston: Fundamentos de Cirurgia. 2 ed. Rio de Janeiro: Guanabara Koogan, 1996.Speranzini, M.e Ramos, M. Manual do Residente de Cirurgia. 3 ed. Rio de Janeiro: Guanabara Koogan, 1988. OUTROS: Prado, F. C. do et al. Atualização terapêutica: manual prático de diagnóstico e tratamento. 21 ed. São Paulo: Artes Médicas, 2005. PÁGINAS DA INTERNET: Sociedade Brasileira de Medicina de Família e Comunidade <www.sbmfc.org.br $>$; Free Medical Journals. <http://www.freemedicaljournals.com/htm>; Bireme $<$ http:/ / www.bireme.br >; Cochrane Collaboration no Brasil <http:/ / www.unifesp.br/cochrane>; National Library of Medicine http:/ / www.nlm.nih.gov/; Medline <http://www.ncbi.nlm.nih.gov:80/ entrez/query.fcgi>; Portal do PSF <http:// www.psfbrasil.com.br >; DATASUS $<$ http:/ /www.datasus.gov.br $>$. 


\section{DA AVALIAÇÃO}

A avaliação é parte inseparável do processo docente-educativo, como retroalimentação da aquisição do conhecimento e como controle do cumprimento dos objetivos, e incluirá aspectos teóricos e práticos. Não deverá atender ao propósito punitivo, mas, sim, ser um meio para o progresso da formação do residente. As avaliações serão: periódicas, durante as atividades diárias; parciais, por meio de prova trimestral; finais, ao término do primeiro e segundo anos. Na conclusão do curso, o residente receberá uma avaliação final que considere suas atividades educativas, instrutivas, assistenciais, éticas e de pesquisa, integrando as avaliações periódicas, parciais efinais comoum todo,nãoum somatório, mas como um resultado integral e progressivo ${ }^{42,43}$. No dizer de Gonçalves ${ }^{43}$, a avaliação deve ser permanente, transparente, judiciosa, construtiva, diagnóstica, gratificante, coerente, prática e global.

\section{COMENTÁRIO FINAL}

Este programa propõe-se a contribuir na formação do MoFC chamado a solucionar os problemas de saúde da população surgidos em conseqüência da excessiva especialização médica. Parte-se da premissa de que a formaçãona Residência Médica (RM) não deve ficar ao livre arbítrio, mas, sim, deve ser embasada numa estratégia que abranja os conhecimentos teórico-práticos de forma integral, geral, atual, sistemática, esquemática, flexível, reproduzível e que permita sua adequação às necessidades das diferentes comunidades, atendendo às indicações e recomendações das instâncias normativas ${ }^{20,21}$. Trata-se de assumir a RM como uma forma de ensino de pós-graduação, na qual o médico recém-formado vai se aperfeiçoar no exercício da profissão, em serviços capacitados e sob a orientação de professores e outros médicos de reconhecida competência ${ }^{44}$.

A proposta do PRMFC apresentada contempla a formação do residente em dois anos, por meio do treinamento supervisionado pelos PP em serviço na US e no HR, onde se procura que alcance os saberes educativos, preventivos, curativos e investigativos em relação ao processo saúde-enfermidade que lhe permitam desenvolver-se de maneira plena e competente no exercício de sua profissão. Além disso, propõe inserir a formação do MoFC no PSF e, assim, entrelaçar e complementar o programa formativo ao assistencial.

Para concretizar esta proposta, é necessário um coordenador especialista em MFC, além de um convênio entre a instituição mantenedora do PRMFC e a Secretaria Municipal de Saúde, atendendo às orientações da CNRM, da SBMFC e do MS. Para sua implantação, propõe-se objetivar as idéias contidas na Figura 1.

Enfim, acreditamos que a proposta colabore para uma concepção do MoFC que atenda às necessidades majoritárias e gerais de saúde.

\section{FIGURA 1}

Esquema docente-assistencial do Programa de Residente de Medicina de Família e Comunidade (PSF: Programa de Saúde da Família do Ministério de Saúde15; MS: Ministério de Saúde5; CNRM: Comissão Nacional de Residência Médica ${ }^{20}$; SBMFC: Sociedade Brasileira de Medicina de Família e Comunidade $\left.{ }^{21}\right)$.

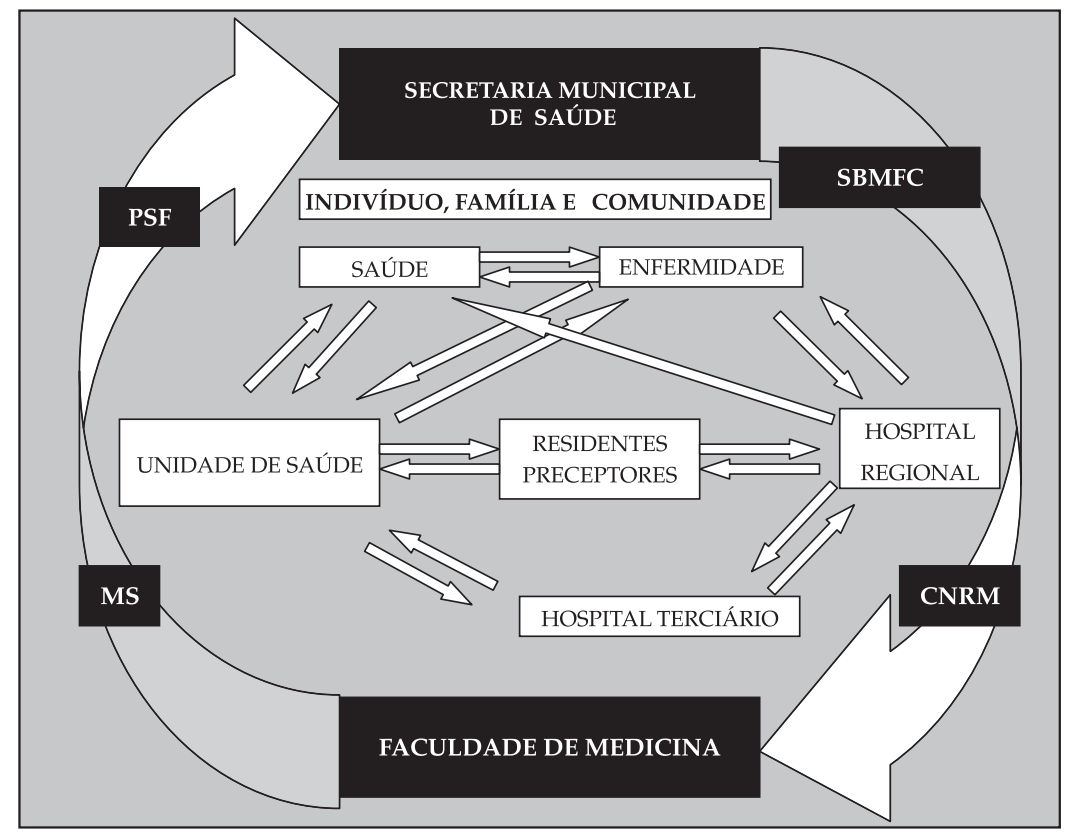




\section{QUADRO 1}

Distribuição das atividades práticas do Residente de Medicina de Família e Comunidade

\begin{tabular}{|c|c|c|}
\hline & Tempo/Carga Horária & Anual \\
\hline \multicolumn{3}{|l|}{ Primeiro ano } \\
\hline Familiarização & 4 semanas $(40 \mathrm{~h} / \mathrm{sem})$ & $160 \mathrm{~h}$ \\
\hline Atenção primária (Unidade de Saúde) & 48 semanas $(40 \mathrm{~h} / \mathrm{sem})$ & $1.760 \mathrm{~h}$ \\
\hline Área/Planejamento & $12 \mathrm{~h} /$ semana & \\
\hline Consultório & $28 \mathrm{~h} /$ semana & \\
\hline \multirow[t]{2}{*}{ Plantões no Hospital Regional } & 48 seman as $(12 \mathrm{~h} / \mathrm{sem})$ & $576 \mathrm{~h}$ \\
\hline & Subtotal & $2.496 \mathrm{~h}$ \\
\hline \multicolumn{3}{|l|}{ Segundo ano } \\
\hline Atenção secundária (Hospital Regional) & 44 semanas $(20 \mathrm{~h} / \mathrm{sem})$ & $880 \mathrm{~h}$ \\
\hline Clínica Médica & 13 semanas $(20 \mathrm{~h} / \mathrm{sem})$ & $260 \mathrm{~h}$ \\
\hline Pediatria & 13 semanas $(20 \mathrm{~h} / \mathrm{sem})$ & $260 \mathrm{~h}$ \\
\hline Ginecologia-Obstetrícia & 13 semanas $(20 \mathrm{~h} / \mathrm{sem})$ & $260 \mathrm{~h}$ \\
\hline Cirurgia Geral & 5 semanas $(20 \mathrm{~h} / \mathrm{sem})$ & $100 \mathrm{~h}$ \\
\hline \multicolumn{3}{|l|}{ Atenção primária } \\
\hline Área/Planejamento & 44 semanas $(8 \mathrm{~h} / \mathrm{sem})$ & $352 \mathrm{~h}$ \\
\hline Consultório & 44 semanas $(12 \mathrm{~h} / \mathrm{sem})$ & $528 \mathrm{~h}$ \\
\hline Eletivo & 4 semanas $(40 \mathrm{~h} / \mathrm{sem})$ & $160 \mathrm{~h}$ \\
\hline \multirow[t]{3}{*}{ Plantões no Hospital Regional } & 44 semanas $(12 \mathrm{~h} / \mathrm{sem})$ & $528 \mathrm{~h}$ \\
\hline & Subtotal & $2.428 \mathrm{~h}$ \\
\hline & Total & $4.924 \mathrm{~h}$ \\
\hline
\end{tabular}

QUADRO 2

Distribuição das atividades teórico-práticas do Residente de Medicina de Família e Comunidade

\begin{tabular}{|c|c|c|}
\hline Atividade & Freqüência & Duração \\
\hline \multicolumn{3}{|l|}{ Medicina de Família e Comunidade } \\
\hline Seminário & Semanal & $1 \mathrm{~h}$ \\
\hline MesaRedonda & Mensal & $2 \mathrm{~h}$ \\
\hline \multicolumn{3}{|l|}{ Clínica } \\
\hline Seminário & Semanal & $1 \mathrm{~h}$ \\
\hline Mesa-Redonda & Mensal & $2 \mathrm{~h}$ \\
\hline \multicolumn{3}{|l|}{ Pediatria } \\
\hline Seminário & Semanal & $1 \mathrm{~h}$ \\
\hline Mesa-Redonda & Mensal & $2 \mathrm{~h}$ \\
\hline \multicolumn{3}{|l|}{ Ginecologia Obstetrícia } \\
\hline Seminário & Semanal & $1 \mathrm{~h}$ \\
\hline MesaRedonda & Mensal & $2 \mathrm{~h}$ \\
\hline \multicolumn{3}{|l|}{ Cirurgia } \\
\hline Seminário & Quinzenal & $1 \mathrm{~h}$ \\
\hline Mesaredonda & Bimestral & $2 \mathrm{~h}$ \\
\hline Reuniões Magnas & Mensal & $2 \mathrm{~h}$ \\
\hline Pesquisa & Mensal & $4 \mathrm{~h}$ \\
\hline
\end{tabular}




\section{REFERÊNCIAS}

1. Lopes OC. A Medicina no Tempo. São Paulo: Melhoramentos; 1970.

2. Capra F. O ponto de mutação. São Paulo: Cultrix; 1982.

3. Aguiar AC de. Tendências na educação médica: um modelo curricular ajustado ao conceito ampliado de saúde, às mudanças tecnológicas e à realidade social. In: Arruda BKG de (Org.). A educação profissional em saúde e a realidade social. Recife: IMIP e Ministério da Saúde, 2001. p. 69-86.

4. Lieberman III JA, Stuart MR. Practing biopsychosocial Medicine. In: Rakel G. Textbook of Family Practice. 6 ed. Philadelphia: 2002, p 65-70.

5. Costa Neto MM. Tecnologia e saúde: causa e efeito? In: Garrafa V, Costa SIF. A bioética no século XXI. Brasília: UnB, 2000. p. 145-150.

6. Martins C. Perspectiva da Relação Médico-Paciente. 2 ed. Porto Alegre: Editora Artes Médicas; 1981.

7. Iberti TJ. American Medical Education. Has It Create a Frankenstein?. Am J Med. 1985; 78 (2): 179-181.

8. Rich EC, Croeson TW, Harris IB. The Diagnostic Value of Medical History. Arch Intern Med. 1987; 147:1957-60.

9. Jinich H. Triunfos y fracasos de la medicina en los Estados Unidos da America. Gaceta Medica de Mexico. 1990; 126 (2) : 72-6.

10. Assad JE. Relação médico-paciente no final do século XX. In: Desafios éticos. Brasília: Conselho Federal de Medicina 1993: 104-111.

11. Rodríguez MAM. Crisis del Método Clínico. Rev Cubana Med, 1998; 37 (2):123-8.

12. Llanio NR, Fernández Mirabal JE, Fernández Sacasa JA. Historia Clinica: La mejor arma del médico en el diagnóstico de las enfermedades. Ciudad de la Habana: Editorial Pueblo y Educación; 1989.

13. Cleaveland CR. Gloom in the trenches. Health Management Quarterly, vol. 15, p. 7-11, 1993.

14. OMS/WONCA (Organização Mundial de Saúde / Organização Mundial dos Médicos de Família). Tornar a prática e a educação médica mais relevantes para as necessidades das pessoas: A contribuição do Médico de Família. Ed Instituto de Clínica Geral da Zona Sul. Ontário: OMS/ WONCA, 1994.

15. Ministério de Saúde. Programa de Saúde da Família. Brasília. Saúde Dentro de Casa. Fundação Nacional de Saúde, Ministério da Saúde. 1994.
16. Machado MH. Pedrosa Neto AH. Carvalho, PRR. Perfil dos médicos. Dados, 19. FIOCRUZ-RADIS. Rio de Janeiro 1996(19).

17. Feuerwerker LMC. A formação de médicos especialistas e a residência no Brasil. Saúde em Debate. São Paulo $2001 ; 25$ : 39-54.

18. Ciampo LAD. Ricco RG, Daneluzzi JC. Residência Médica em Medicina Geral e Comunitária. Proposta de um Programa de Formação de Médicos Generalista. Rev Bra Educ Méd. Rio de Janeiro 2003; 27(3): 200-204.

19. Sociedade Brasileira de Medicina de Família e Comunidade. Relação das Residências de Medicina da Família e Comunidade [documento da Internet]. SBMFC, 2004 [acessado em 05 de setembro de 2005] http:// www.sbmfc.org.br/site/capacita/residencias.pdf.

20. Secretaria De Educação Superior, Comissão Nacional de Residência Médica. Resolução no 1. Brasília: CNRM, 14 de maio de 2002.

21. Sociedade Brasileira de Medicina de Família e Comunidade. Projeto de expansão da Residência de Medicina de Família e Comunidade [documento da Internet]. SBMFC, 2005 [acessado em 26 de julho de 2005] http:/ /www.sbmfc.org.br/site/not/download/ Projeto de Expansão dos PRMFC-SBMFC.pdf.

22. Ruiz JÁ. Como elaborar trabalhos de pesquisa. In: Metodologia científica: Guia para eficiência nos estudos. São Paulo: Atlas, 1988, p. 48-84.

23. Severino AJ. Metodologia do trabalho científico. São Paulo: Cortez, 2000.

24. World Health Organization. Reorienting Medical Education and Medical Practice for Health for All. The forty-eight World Health Assembly, South Africa, 1995. Publican in Education for Health. 1996, vol. 9, n. 1, p. 5-11.

25. Almeida, MJ. Educação médica e saúde: possibilidades de mudança. Londrina: UEL. Rio de Janeiro: ABEM, 1999.

26. Almeida MJ. Tecnologia e medicina: uma visão da Academia. Bioética: Brasília, vol. 8, n.1, p.69-78, 2000.

27. Ferreira Filho OF. et al. Visão docente do processo de implementação da aprendizagem baseada em problemas (ABP) no curso médico da Universidade Estatual de Londrina. Rev Bras Educ Med. Rio de Janeiro, vol. 26, n. 3,p.175-183, 2002.

28. Freire P. Pedagogia da Autonomia: Saberes necessário à prática educativa. 29 ed. São Paulo: Paz e Terra; 1999.

29. Veiga IPA et al. Aula universitária e inovação. In: Veiga IPA, Castanho, MELM (Org.) Caminhos da 
profissionalização do magistério. Campinas: Papirus, 2000. p. 189-190.

30. Morin E. A cabeça bem-feita: repensar a forma, reformar o pensamento. 4 ed. Rio de Janeiro: Bertrand Brasil; 2001.

31. Stine C, Kohrs FP, Little DN, Kaprielian V, Gatipon BB, Haq C. Integrating Prevention Education into the Medical School Curriculum: The Role of Departments of Family Medicine. Acad Med. 2000; 75: 55-9.

32. Steele D, Susman J, Mccurdy F, O'dell, Paulman P, Stott J. The Interdisciplinary Generalist Project at the University of Nebraska Medical Center. Acad Med. 2001; 76: 121-6.

33. Lopez Santiago A, Baeza Lopez JM, Lebredo Garcia RM. Qué esperan de sus tutores los residentes de Medicina de Familia? Una aproximación cualitativa. Aten Primaria. 2000; 26(6):362-7.

34. Rodríguez CA. As inovações no ensino superior e a formação do médico professor. 2003. 146f. Tese (Mestrado) _C Curso de Pós Graduação do Centro de Teologia e Ciências Humanas. Pontifícia Universidade Católica do Paraná, Curitiba.

35. Starfield B. Atenção primária. Brasília: UNESCO, 2002.

36. DeHaven MJ, Wilson GR, Murphee DD, Grunding JP. Family practice residency program directors' views on research. Fam Med. 1997:29: 33-37.

37. Carek PJ. Being successful with family medicine residency research: lessons learned from others. Ann Fam Med, v. 1, n. 4, nov-dec 2003: p. 246.

38. Rodriguez CA, Strozzi JB, Cassias AL. Programa da Residência de Medicina e Comunidade PUC PR. Curitiba: Editora Champagnat; 2001.
39. Vernon DTA, Blake RL. Does problem-based learning work? A meta analisis of avaluative research. Acad Med. 1993; 68: 550-63.

40. Kaufman DM, Mann KV. Comparing students attitudes in Problem-based and Conventional Curricula. Academic Medicine. 1996; 71:1096-9.

41. Sackett DL, Straus SE, Richardson WS. Evidence-Based Medicine: How to Practice and Teach, 2a ed. San Antonio : Churchill Livingstone, 2000.

42. Venturelli J. La evaluación em el proceso de formación de los profesionales médicos: princípios de evaliación formativa. In: __. Educación Médica: Nuevos enfoques, metas y métodos. Washington, D.C.: Organización Panamericana de la Salud, 1997. p. 155-179.

43. Gonçalves EL. Três Escolas Médicas Numa Escola. In: Marcondes E, Gonçalves EL. Educação Médica. São Paulo: Sarvier; 1998. p. 355-68.

44. Gonçalves EL. Médicos e Ensino de Medicina no Brasil. São Paulo: EDUSP, 2002.

\section{CONFLITOS DE INTERESSE}

Declarou não haver.

\section{ENDEREÇO PARA CORRESPONDÊNCIA}

Carlos Arteaga Rodríguez

Rua Oyapock, 367 - Cristo Rei

80050-0450 - Curitiba - Paraná

E-mail: arteaga.carlos@pucpr.br; arteagarodriguez@hotmail.com 